\title{
Discurso De Los Estudiantes De La Licenciatura En Educación Especial, Sobre La Formación Docente. Escuela Normal Fray Matías De Córdova. Tapachula, Chiapas
}

\author{
Speech Of Undergraduate Students In Special Education Teacher \\ Training On. Basic School Fray Matías Cordova. Tapachula, Chiapas
}

Mauricio Zacarías Gutiérrez¹, Ma. Juana Eva Luna Denicia², José Macías Equihua³

- Mauricio Zacarías Gutiérrez. Docente-investigador de la escuela Normal "Fray Matías de Córdova" Tapachula, Chiapas. México mazag@hotmail.com

2 - Ma. Juana Eva Luna Denicia. Docente-investigadora y sub-directora académica de la escuela Normal "Fray Matías de Córdova" Tapachula, Chiapas. México.evacarton@hotmail.com

José Macías Equihua Docente-investigador y director de la escuela Normal "Fray Matías de Córdova" Tapachula, Chiapas. México. Maciasjoe3@hotmail.com

Resumen

El presente trabajo, es parte de la investigación Perspectiva de los alumnos respecto a la formación como licenciados en Educación Especial. Llevada a cabo en el municipio de Tapachula, Chiapas. México. El propósito del artículo es dar cuenta del discurso que tienen los estudiantes respecto a su formación docente inicial en Educación Especial. El supuesto en el que se fincó la investigación es que a los alumnos se les ha formado con dominios teóricos y prácticos para enfrentar la realidad educativa en el contexto de su ejercicio profesional. La posición epistemológica de la investigación es el cualitativo, usando como método y técnica de estudio el grupo de discusión. Finalmente, comentar que los resultados de la investigación que aquí se presentan es un avance de resultados.

Palabras claves: discurso, educación especial, formación

68 GUTIÉRREZ, M.Z.; DENICIA, M.J.E.L.; EQUIHUA, J.M.: Discurso De Los Estudiantes De La Licenciatura En Educación Especial, Sobre La Formación Docente. Escuela Normal Fray Matías De Córdova. Tapachula, Chiapas. 


\begin{abstract}
This work is part of the student research perspective regarding training as graduates in Special Education. Held in the city of Tapachula, Chiapas. Mexico. The purpose of this paper is to account for the speech that students have regarding their initial teacher training in special education. The case in which the investigation was Finch is that students have formed them with theoretical and practical domains to meet the educational reality in the context of their professional practice. The epistemological position is the qualitative research as measured technique study and discussion group. Finally, commenting that the results of the research presented here is a preview of results.
\end{abstract}

Keywords: speech, special education, training

\section{Introducción}

En el proceso de indagación sobre qué se ha escrito en el ámbito de la formación inicial docente en educacion especial, llevó en primera instancia a revisar investigaciones que hayan realizado las escuelas normales que forman a estos alumnos, sin embargo, no se encontró investigación alguna. Solo se encuentra el plan de estudio, que es el rector institucional de la formacion inicial docente de la licenciatura. Cabe decir que el plan de estudio es homogeno, es planteado por la Secretaría de Educación Pública, y con ese se forma en las escuelas normales.

Dentro de los rasgos deseables que establece el plan y programa de estudio 2004 para el licenciado en la Educación Especial se encuentran los siguientes: "habilidades intelectuales específicas; conocimiento de los propósitos, enfoques y contenidos de la educación básica; competencias didácticas; identidad profesional y ética; y capacidad de percepción y respuesta a las condiciones sociales de sus alumnos y del entorno de la escuela" (Secretaría de Educación Pública, 2004, p. 44). Precisando el rasgo deseable de identidad profesional y ética, se consideran los siguientes campos a desarrollar en el alumno en el proceso de la formación en la escuela normal. A continuación, se enlistan solo uno de los elementos del rasgo de formación Identidad profesional y ética.

a) Asume como principios de su acción y de sus relaciones con la comunidad educativa, los valores que la humanidad ha creado y consagrado a lo largo de la historia: respeto y aprecio a la dignidad humana, libertad, justicia, igualdad, equidad, democracia, solidaridad, aceptación, tolerancia, honestidad, responsabilidad y apego a la legalidad.

d) Identifica y valora los elementos más importantes de la tradición 
educativa mexicana; en particular, reconoce cómo ha evolucionado la educación especial en nuestro país y la importancia de la educación pública como componente esencial de una política basada en la justicia, la democracia y la equidad.

e) Se compromete con la misión y los principios de la educación especial, y reconoce la importancia de brindar atención educativa a los alumnos que presentan necesidades educativas especiales, con o sin discapacidad, para lograr el desarrollo integral de sus potencialidades y favorecer su integración social y laboral.

\section{1 - Campos De Formación}

Estos campos de formación en los alumnos mantienen relación con los otros rasgos, sin embargo, para los fines de la investigación que se realiza se precisan estos, dado que llevan la orientación de la formación docente. En el proceso de indagación sobre qué se ha escrito respecto a la formación inicial docente, hay investigaciones que plantean cómo se ha dado el proceso. Los enfoques metodológicos de las investigaciones en el abordaje de la formación han sido diferentes, sin embargo, todas coinciden en el paradigma cualitativo. En tal caso, las metodologías en que ha sido abordado cada objeto de estudio, da elementos para plantear el objeto de esta investigación. La investigación de Barba (2010), plantea un análisis de la valoración de la reforma de la formación docente por profesores con diferentes funciones en el estado de Aguascalientes. La investigación estuvo enfocada en el análisis de los rasgos positivos de la reforma educativa de la licenciatura en educación primaria en 1997, así como sus logros y obstáculos para su implementación.

Plantea Barba que:

El centralismo político-educacional del país, con la dependencia financiera y operativa asociadas, es un factor que no da aliento a que en los estados se desarrolle de modo suficiente la competencia gubernamental y de gestión en la formación inicial de docentes que la sociedad necesita, pero esto no exculpa a las autoridades estatales (2010, p. 271).

Los planteamientos a los que llega Barba, permite identificar que la manera en que los profesores valorizan la reforma educativa en la formación inicial docente en la educación primaria requiere que se considere desde ellos, son ellos quienes pueden favorecer un plan de estudio que articule sus necesidades al contexto Chiapas. 
en el que se encuentran.

En otra perspectiva la investigación de Furlan (2005), explora los cambios que ha habido entre la formación de docentes de nivel secundaria que se propusieron en el plan de estudios de 1999 y algunas secundarias del distrito federal relacionado a la problemática de indisciplina y/o violencia. Ello en una relación entre la materia de Atención educativa a los adolescentes en situaciones de riesgo en cuanto a la problemática mencionada y las actividades de observación y práctica docente en la formación de profesores.

Plantea Furlan que "la propia estructura del currículum dificulta el desarrollo de miradas integradoras y críticas" (2005, p. 1101), dado que hay una formación segmentada en las escuelas normales que desalienta el trabajo en equipos multidisciplinarios. Se reconoce que en las jornadas de observación y práctica docente hay una separación entre la instrucción que competencia del practicante y la gestión de conductas disruptivas, que es propio de la escuela donde practican. Ello dice Furlan que "provoca una mutilación del trabajo docente real" (2005, p. 1102). Sitúa que se requiere un planteamiento curricular que considere la formación de los alumnos donde intervenga positivamente con aspectos vinculados a la socialización y desarrollo de la personalidad de los alumnos.

La investigación de Farías Martínez y
Ramírez Montoya, (2010), da cuenta de las cualidades reflexivas que desarrollan o fortalecen profesores en formación al hacer portafolios electrónicos de trabajo, que no se corresponde con el diario de campo del profesor que habitualmente se usa en la escuela normal para que los alumnos vayan registrando sus observaciones de las clases. Consideran los autores que

Los resultados obtenidos muestran que la construcción de un portafolio electrónico de evidencias, dentro de un entorno colaborativo, favorece la reflexión en los estudiantes, pues se detectaron diferencias particulares en el desarrollo - fortalecimiento de las cualidades reflexivas propias de la dimensión interpersonal, entre los alumnos que utilizaron portafolios electrónicos de evidencias y los que no lo utilizaron (2010, p. 155).

Desde la postura de los autores, aun falta por profundizar en la utilización de estrategias que fortalezcan la formación inicial de los docentes que se enuncian en los perfiles de egreso del estudiante normalista. Ello considera que la formación docente inicial considere como dimensiones tanto de recursos que se les da a los normalistas para que reflexionen su práctica, como otros elementos (el portafolio electrónico de evidencias), donde se tenga en cuenta cómo se piensa la formación docente.

A partir de las revisiones de 
investigaciones realizadas y del perfil de egreso de la licenciatura en Educación Especial, permite aclarar el objeto de estudio de la investigación. Por lo que emergió las siguientes interrogantes. ¿Cuál es el discurso que tienen de la formación inicial en la educación especial, los alumnos de la licenciatura en Educación Especial, de la escuela normal "Fray Matías de Córdova"? Tapachula, Chiapas? Ante ello planteamos el problema de investigación.

\section{2 - Problema De Investigacion}

En el año 2004 inicia a funcionar la Licenciatura en Educación Especial en la escuela normal "Fray Matías de Córdova", con dos Áreas
Específicas: Área Intelectual y Área de audición y Lenguaje. Teniendo para ello diferentes campos de formación.

En la escuela normal "Fray Matías de Córdova" en el 2008 egresó la primera generación de Licenciados en Educación Especial. A la fecha han salido 8 generaciones de las cuales ya se encuentran en servicio docente. En la convocatoria para obtener una plaza docente, para el ciclo escolar 2014-2015, la escuela normal ocupó un buen posicionamiento de acuerdo a la cantidad de alumnos que egresaron y que obtuvieron una plaza docente. La gráfica 1, da cuenta del porcentaje de alumnos que obtuvieron una plaza docente, vía concurso.

Gráfica 1. Desempeño de alumnos de la escuela normal "Fray Matías de Córdova" a partir del examen de oposición de plazas docentes.

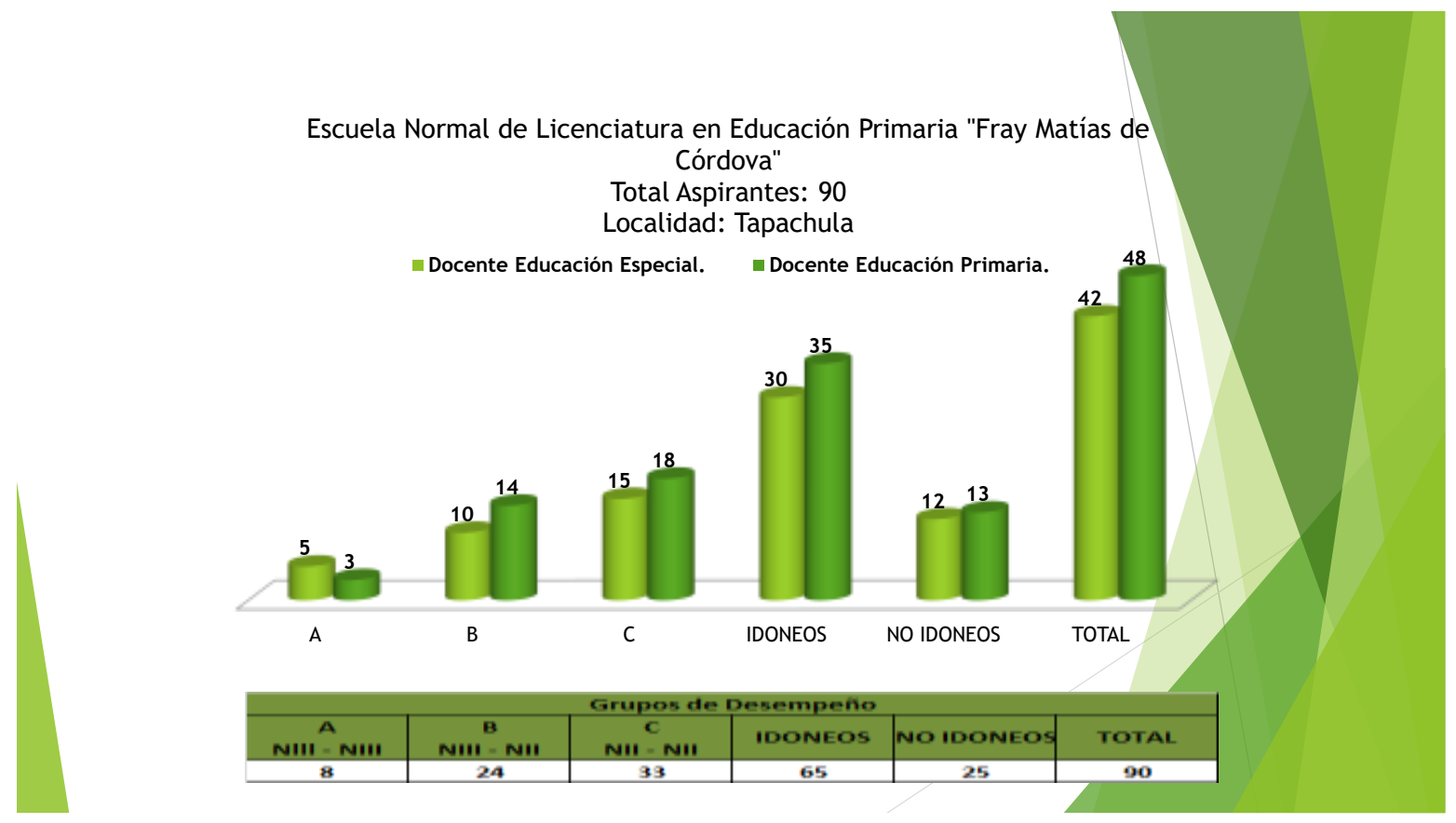

72 GUTIÉRREZ, M.Z.; DENICIA, M.J.E.L.; EQUIHUA, J.M.: Discurso De Los Estudiantes De La Licenciatura En Educación Especial, Sobre La Formación Docente. Escuela Normal Fray Matías De Córdova. Tapachula, Chiapas. 
Fuente: Tomado del Instituto Estatal de Evaluación e Innovación Educativa. Dirección de Evaluación

Educativa.

De los 90 alumnos que egresaron, el $72.2 \%$ salió idóneo y el $27.8 \%$ no idóneo. Ello incluye el total de alumnos que egresaron tanto de la licenciatura en Educación Primaria como en Educación Especial, que son las dos licenciaturas que se imparten en la escuela normal "Fray Matías de Córdova". En cuanto a la licenciatura en Educación Especial, 71.4\% salieron idóneos y $28.6 \%$ no idóneos, ello permite inferir que la escuela normal en la licenciatura tiene una regular formación en los alumnos que forma para su ingreso al servicio docente.

Con base al planteamiento anterior, la séptima generación egresada en el año 2014 al presentar examen de oposición para obtener su plaza, la mayoría lo obtuvo. En este año 2015 realizarán la evaluación diagnóstica convocada por el Instituto Nacional para la Evaluación de la Educación, que valorará su desarrollo docente ejercido en el ciclo escolar 2014-2015.

Independiente a la evaluación diagnóstica que presentará la generación egresada en el 20142015, y sabidos que para el ciclo escolar 20162017 inicia una nueva licenciatura en la escuela normal titulada Licenciatura en Educación Inclusiva, es pertienente realizar el estudio sobre la perspectiva de formación en estos alumnos, dado que hasta el ciclo 2018-2019 se cierre la licenciatura en Educación Especial.
Ello lleva a precisar el problema de investigación en torno al discurso sobre la formación docente inicial en educación especial. El objetivo de la investigación se centro en analizar ese discurso.

\section{3 - Supuesto Hipotético}

La perspectiva que tienen de la formación inicial en la educación especial, los alumnos de la licenciatura en Educación Especial, de la escuela normal "Fray Matías de Córdova". Tapachula, Chiapas, es que se les ha formado con dominios teóricos y prácticas para enfrentar la realidad educativa en el contexto de su ejercicio profesional.

Metodología: La investigación se ubica en la estrategia metodológica cualitativa, toma como método de estudio la técnica de grupo de discusión, apoyándose con la aplicación de un cuestionario para triangular información respecto a la obtención de información sobre opiniones, percepciones e intereses de pequeños grupos individuales (HUERTAS-BARROS Y VIGIERMORENO, 2010).

Ibáñez (2003), plantea que en el grupo de discusión los investigadores se asumen como seres en proceso, en el proceso de la investigación. Las personas que participan en el grupo se plantea deben ser homogéneas, de tal manera que el investigador sea el heterogéneo en 
el mismo. La cantidad de personas que participan serán hasta tres cómo mínimo dice Ibáñez, hasta 12 como máximo.

En el caso de la investigación, participaron alumnos que se forman en la licenciatura en Educación Especial, de un total de 104 alumnos inscritos en los cuatro semestres, solo participaran en los grupos de discusión dos estudiantes por semestre. Ello involucra considerar que será homogéneo el grupo, dado que cursan la misma licenciatura. Los criterios que se considerarán para elegir a los estudiantes son los siguientes.

- Uno de ellos que provenga de padres profesionistas (los dos padres o al menos uno) y el otro que sus padres no sean profesionistas.

- Que tenga un promedio entre 8.0 a 8.5, en el semestre que cursan.

- Tener el 100\% de asistencia.
Marco teorico: La formación docente varía de país a país, y mantiene intereses centrados en el desarrollo económico del mismo. Así podemos leer en el texto de Tardif (2004) la manera en que han sido enfocadas las reformas educativas en la formación de los docentes en Estados Unidos y Canadá. En el planteamiento que tiene el autor sale avante la función de la universidad y su relación con el docente en servicio. El que llega todos los días al aula y forma desde su saber que ha construido en la racionalidad que le da la práctica.

En el caso de México, hasta el 2013 las escuelas normales eran las únicas que aportaban docentes al sistema educativo, a partir de la reforma educativa constitucionalmente establecida en diciembre de 2013, se da apertura a que universitarios puedan aspirar a ingresar al servicio profesional docente. Ello cuestiona la dinámica en que las normales se encuentra y cual es el rumbo al que se les enfoca, una vez que no tienen constitucionalmente la autonomía que tienen las universidades, por consiguiente no son autónomas en sus decisiones, dependiendo de la Secretaría de Educación Pública en sus decisiones y en la formación de los docentes iniciales que atienden la educación básica en el sistema educativo mexicano.

La formación inicial docente, por tanto en México se mantiene entre las escuelas normales formadoras de docentes y las universidades. Lo

74 GUTIÉRREZ, M.Z.; DENICIA, M.J.E.L.; EQUIHUA, J.M.: Discurso De Los Estudiantes De La Licenciatura En Educación Especial, Sobre La Formación Docente. Escuela Normal Fray Matías De Córdova. Tapachula, Chiapas. 
particular en las escuelas normales es que los docentes en formación realizan prácticas docentes en planteles de la educación básica. Tardif, al respecto señala "la formación inicial pretende habituar a los alumnos -los futuros profesores- a la práctica profesional de los docentes y a hacer de ellos prácticos "reflexivos" (2004, p. 213).

Retomando esta idea, esa es la función en México, de los docentes en formación en las escuelas normales. En cada uno de los semestres los alumnos de cualquier licenciatura que estudie en una escuela normal, realizan al inicio de la carrera un reconocimiento de la práctica docente, $\mathrm{y}$ en el avance de los semestres se involucran paulatinamente a la práctica de la enseñanza de los contenidos y del control del grupo, o como plantea Tardif, a dar a conocer el conocimiento y a ser gestor dentro del aula.

La manera en que se forma a los docentes iniciales en las escuelas normales, difiere de la formación de quien se educa en las universidades, donde el centro de la formación si bien tiene orientaciones pedagógicas y didácticas el enfoque se centra en la discusión teórica. En el caso de las normales hay una praxis, en el entendido de que combina teoría y práctica, de tal manera que el profesor en formación se suministra de teoría y de práctica para realizar el ejercicio de la docencia al término de la formación en la escuela normal.

En este sentido, cuando se habla de la formación inicial de los docentes se considera que deben estar preparados para atender un currículo, la descripción que hace Tardif sobre el profesor técnico, disciplinar e interactivo permite ilustrar el tipo de profesor que se forma en las escuelas normales en México, dado que los programas de estudio para atender la educación básica plantean un docente que trabaje el plan de estudio, que gestione un ambiente de armonía en el aula y que interactué con alumnos, padres, maestros, comunidad, entre otros. Un profesor que se vuelva aprendiz de su propia práctica y se proyecte al exterior como intelectual de la enseñanza.

Gimeno Sacristán (2001) al respecto plantea la formación de un ciudadano, que no se limite a su territorio, sino que considere los contextos globales en que se sitúa la práctica docente que realiza. No solo se voltea la mirada hacia la formación instrumental del sujeto, sino que se considera a un sujeto que comparte una tierra patria como plantea Morín (2010), de tal manera que la formación inicial del profesorado considere al sujeto en la condición en la que se encuentra, sin obviar el contexto global en el que se encuentra inmerso.

Una formación holística en los docentes que no solo considera el plan y programa de estudio con el que se forma y con el que formará, sino que tenga en consideración que tal formación que se reciba no se limita a las maneras mecánicas de la enseñanza. La didáctica como parte inmanente a la formación de los 
profesores lleva inmerso una corriente epistemológica sobre el conocimiento que se tiene que desarrollar en el que se forma. En este caso, el pensamiento cartesiano es el que abandera la formación docente, en el sentido de que se busca el desarrollo de estado-nación a través de la educación, siendo los docentes quienes tienen que desarrollar esos conocimientos en los alumnos que forman.

Para ello la formación a la que responden ha dejado de ser a la que se formó después de la posguerra como plantea Day (2005) y se considera que "en particular, lo que aprenden los estudiantes, lo que deben conseguir como resultado del aprendizaje y las normas que se apliquen son ahora asunto cotidiano y explícito del gobierno" (2005, p. 26). Hay una centralidad de qué debe de aprender el que se forma en la docencia. Sobre ello se plantea el interés de qué formar, con qué didáctica orientar la enseñanza, para que cuando esté en el campo con el objeto en formación, el profesional de la enseñanza tenga claro qué se busca de él y qué se busca del estudiante que forma.

Esta claridad de ideas que puede tener el profesional de la enseñanza se complejiza con la responsabilidad moral que adquiere en la formación de los alumnos que atiende, dado que "los docentes no son sólo receptores del cambio político iniciado en el exterior de sus escuelas y aulas, sino que, también, son iniciadores del cambio" (Day, 2005, p. 30). Para ello el profesorado considera que no trabaja con objetos (como hemos mencionado líneas arriba), sino que considera las condiciones en que los alumnos han vivido la moralidad. Ello contextualiza un hacer docente que no solo es técnica, sino moral, a lo que Tardif llama relacional.

No se obvia en este análisis teórico sobre la formación docente que la escuela que forma plantea objetivos de qué enseñar. En este caso, los modelos económicos que tienen los países inciden en la didáctica que ocupan los docentes en la enseñanza que realizan, la cual se traspasa al aprendizaje del estudiante, en este mundo globalizado en el que se vive actualmente.

Trabajos como el Pérez Gómez (1999), Torres (1994), Sacristán (2001) entre otros, han dado cuenta que el modelo económico capitalista ha permeado los currículos escolares con los que se forma en la escuela. La cual da una prioridad relevante a la razón. A partir de este objetivo sobre qué educar y para qué educar en la escuela, se toman a todos los agentes externos que participan en el proceso formativo como objetos complementarios para desarrollar la razón en el estudiante. No se considera la condición social y cognitiva en que se encuentra el alumno para que aprenda, sino que, se establece desde el currículo escolar el enfoque didáctico que tendrá la formación de los alumnos, que particularmente en básica, tal formación es homogénea, que si

76 GUTIÉRREZ, M.Z.; DENICIA, M.J.E.L.; EQUIHUA, J.M.: Discurso De Los Estudiantes De La Licenciatura En Educación Especial, Sobre La Formación Docente. Escuela Normal Fray Matías De Córdova. Tapachula, Chiapas. 
situamos este planteamiento de homogeneización en la educación normal formadora de maestros, hay un enfoque didáctico homogéneo en la formación docente inicial.

Torres (1994), desde la manera en que ha estudiado el currículo sostiene la interdisciplinariedad en la construcción del currículo, hace una distinción entre lo disciplinar, multidisciplinar, interdisciplinar, transdisciplinar postura que es entendible, para comprender el proceso de formación que se da en las escuelas a partir del modelo económico que establecen los países para generarse desarrollo económico.

La manera de enseñar por tanto responde a intereses que como sitúa Ardra Cale (citado en Day, 2005) "la mayoría de las iniciativas aún están "conceptuadas, diseñadas y facilitadas para los docentes y no por ellos" (58). Ello nos refiere que la formación docente cuando se centraliza responde a intereses ajenos a los que forman y a los propios formados, desde el planteamiento de Bourdieu (1996) ello se relaciona a la violencia a la que son sometidos quienes se forman en la docencia, en el sentido de que no solo se involucra qué enseñar y cómo enseñar, sino que tanto la enseñanza como el aprendizaje responden al interés de quien ha diseñado tal formación.

\section{4 - La Ideología En La Formación Docente}

La formación profesional lleva un sello ideológico de para qué se forma a quien se forma en la disciplina, ello lleva explícitamente la profesionalidad del que se forma, las habilidades de investigación que usará para mantener la profesión en la sociedad, los códigos éticos que mantendrá no solo como individuales, sino como manutención de la misma profesión. Hay un mantenimiento del orden en las disciplinas, hay límites en las fronteras de cada disciplina. Como menciona Torres (1994), en algunos caso se traspasan las fronteras, sin embargo cada uno cuida sus particularidades.

La formación docente, se ubica en la disciplina de la pedagogía, a la vez se alimenta de otras disciplinas para poder ser ella. La pedagogía es una disciplina que mantiene frontera, sin embargo, en su interior hay áreas de la disciplina que la mantienen vigente, a la vez hay en cada área una tradición por tomar la idea de Khun, de qué es lo que se tiene que mantener y sobre qué hay que profundizar en el conocimiento para que la disciplina se mantenga. En tal caso, dentro de la formación docente, hay una ideología de para qué se forma en la docencia, Van Dijk (2005) en este caso remite que las ideologías permean en sectores, donde se comparten sistemas de creencias.

\section{Resultados}

$\underline{\text { www.fics.edu.br } 77}$ 
Se presentan tres apartados de los resultados que se han obtenido hasta el momento de la investigación: la formación docente como ideología, la evaluación y las prácticas docentes.

\section{1 - Analisis del discurso respecto de la formación docente como ideología.}

El discurso que tienen los alumnos que se forman como licenciados en educación especial tiene como base un discurso vivido a partir de la educación formal precedente en las escuelas del Sistema Educativo Mexicano. La formación como tal lleva implícito una ideología sobre qué se debe formar en ellos, entendiendo el debe, como esa posibilidad de mejorar las condiciones sociales en que se encuentran. Una condición social que deviene de las mismas condiciones en que viven.

En este sentido se analiza la manera de cómo se pronuncia el deber al que se sujetan como estudiantes de la licenciatura en Educación Especial. En ello va inmersa una carga ideológica respecto al rol a desarrollar en el momento en el que estén dentro de la práctica docente como profesionales de la disciplina.

Con base a lo anterior se construyeron temáticas respecto a la manera que discursan la formación en educación especial. La cual encierra los elementos de teoría y práctica en la formación durante la escolarización, la adecuación curricular para el alumno con el que practican y la manera en que son formados para cubrir el perfil de egreso. Respecto a la teoría-práctica del aula, hay una ideología fraguada en los alumnos del deber que deberían estar apropiándose en la escuela normal. Un deber sobre qué profesores y cómo serían esos profesores para que la formación que reciben sea la adecuada según ellos desde la manera en que ven la realidad en el campo de la practica docente.

"porque de nada nos sirve que solo nos den teoría sino nos dan ejemplos de esa teoría" P1

"pienso que las teorías son importantes cuando los maestros dan a conocer su tema que es lo que vamos hacer o realizar en las practicas docentes, pero bueno yo siento que, hay que enfocarse más tal vez o, que se enfoquen mas los maestros en el tema que nos van a desarrollar para dar a conocer nuestros programas" P2

La manera en que el discurso se plantea por los estudiantes, da cuenta de la necesidad de recuperar y situar una teoría que se acorde a la realidad educativa en la que realizan la práctica docente. El deber encarnado para lo que se les está formando es la idea de que entre mejor dominio de la teoría tenga el profesorado en formación mejor realizará la práctica docente. La

78 gUTIÉRREZ, M.Z.; DENICIA, M.J.E.L.; EQUIHUA, J.M.: Discurso De Los Estudiantes De La Licenciatura En Educación Especial, Sobre La Formación Docente. Escuela Normal Fray Matías De Córdova. Tapachula, Chiapas. 
organización del discurso sitúa la necesidad de que no se dejen vacíos, que la formación que se les provea se refleje en la práctica docente que realicen.

Que los profesores me enseñen bien las teorías, el desenvolvimiento del profesor y el dominio del tema es demasiado importante porque si el maestro no entiende su tema, si el maestro nomas lo leyó y no lo explica cómo quiere que yo lo entienda. P5

En el discurso que se ha ido apropiando de lo que es la formación docente, el estudiante es consciente de que le implica un responsabilidad, que tal responsabilidad debe estar cimentada en las bases teóricas con las que se les forma. Esta manera de entender el estudiante la formación que recibe como el especialista en la docencia en atención a la educación especial, da elementos para considerar que no se puede abandonar una formación profesional desde lo empírico, sino que la base de la teoría es y se hace fundamental en la formación docente.

\section{5 - E1 Deber De Evaluarse}

El discurso que tienen los estudiantes sobre la evaluación en su proceso formativo nos permite entender, que hay en el lenguaje un nosotros desvinculado del lenguaje del otro (del docente). yo creo que son nada más como bien lo dicen criterios de evaluación, pero, que miden en su momento tu nivel cognitivo, pero, no es el que realmente define y dice si tú sabes más o menos, sino que ya es como tú lo puedes poner en práctica pienso. P8

Una evaluación que se hace necesaria en el proceso de formación, la cual mide un conocimiento a partir de criterios que son aceptados por ambas partes, por el estudiante que acepta (muchas veces no tiene otra opción) la manera en que le será objetivado el conocimiento que adquiere en el proceso de formarse, y el docente que impone sus criterios. Criterios que no precisamente devienen de la iniciativa del profesor, sino que hay elementos del sistema que se impone al maestro, haciendo énfasis de que no se valide el conocimiento del estudiante con un solo criterio. En tal caso, el estudiante reconoce que los criterios que el profesor ocupe, son los que determinan cuanto ha aprendido, ello no solo le valida el momento del curso, sino toda la vida.

En ello podemos encontrar la atención que el alumno le presta a esos criterios, en el sentido del compromiso que ejercen para ellos las tareas escolares que se solicitan para que el conocimiento que se adquiere vaya en la línea de agenciarse del conocimiento que el plan y programa de estudio plantea en los estudiantes. Esas acciones que se realizan en el proceso de validar el conocimiento del alumno, lleva inmerso 
el logro de perfiles de egreso planteados en el plan y programa de estudio.

Van Dikj (2005), plantea que en el discurso hay ocultamiento de lo que quieren decir, en este caso, al tomar la idea del estudiante al decir que un criterio de evaluación no es lo que define si tú sabes más o menos, invita a revisar qué quiere el profesor que el alumno aprenda, qué espera que el alumno domine al terminar el proceso formativo como educador de la educación especial, se plantea también ese reconocimiento que la vida social no se circunscribe en los criterios que el profesor determina para validar los conocimientos que los alumnos aprenden en la escuela. Una sociedad que no se determina con criterios sino que se inscribe en normas formales e informales, las primeras referidas a la reglamentación a partir de un consenso político institucional para que las personas se adecuen a lo establecido. ello da orden a la vida en sociedad, y las normas informales se enfocan a la cotidianidad en la que el alumno se involucra, donde lo inesperado, lo común es lo que da sentido el vivir en la sociedad.

Esta postura se refleja en el discurso de evaluación que tiene el alumno sobre la manera en que se le asigna un número y la diferencia que encuentra con los conocimientos que construye y re-construye en el proceso de la práctica y de las reflexiones que hace la formación, ello da soporte al discurso de dominio público que se aprende con, sin y a pesar del maestro.

\section{6 - La Práctica Docente Realizada Cada Semestre}

En el proceso de la evaluación el alumno va conceptualizando qué implicaciones tiene para él formarse como profesionista en la educación especial. Diferencia claramente que en las jornadas de prácticas docentes que realiza, hay abandono de los especialistas sobre lo que él hace. $\mathrm{Al}$ respecto los estudiantes comentan.

Nosotros pedimos que nos ayuden, a poder, también que nos revisen el plan de trabajo y las adecuaciones, para que el alumno pueda, podamos enseñar bien, pues, pero a veces la docente de educación especial de USAER, pues no nos apoya, entonces no podemos realizar una buena práctica con el niño. P6

El maestro de USAER cuando estás, llegas a practicar con todo el grupo y tienes niños con necesidad educativa, hay algunos que en realidad no te apoyan, ni siquiera te van a observar, no observan tu trabajo. Igual el maestro frente a aula no, no lo hace, y a veces te califica de que no llevas material y las adecuaciones necesarias. A veces tienes dos niños con discapacidad intelectual digamos en un

80 gUtIÉRREZ, M.Z.; DENICIA, M.J.E.L.; EQUIHUA, J.M.: Discurso De Los Estudiantes De La Licenciatura En Educación Especial, Sobre La Formación Docente. Escuela Normal Fray Matías De Córdova. Tapachula, Chiapas. 
salón, pero porque no es la misma discapacidad, no va a tener la misma adecuación, porque ya hay algunos que ya te saben contar del uno al 10, hay otros que ni siquiera te saben contar eso, entonces tienes que hacer la adecuación por cada alumno, no en general. P7

Los discursos que hacen las alumnas respecto a la ausencia en la formación de la práctica docente en las Unidades de Servicios de Ayuda Especial en el aula Regular (USAER), permite reconocer dos situaciones: por un lado un dejar ser en la práctica docente para el estudiante. Que el reflexione si lo que hace es lo correcto de acuerdo a la formación teórica recibida en la escuela normal, pro el otro, el deseo del alumno que se le diga si es correcto lo que hace en el tiempo que realiza la práctica.

El estudiante refleja el deseo de que se le corrija la acción que realiza en el periodo de tiempo que él se encarga del grupo de estudiantes que le responsabilizan durante la práctica docente. En el discurso que sostiene el estudiante, se comprende una ideología enfocada al deber, un deber que le garantice que si lo que realiza es lo correcto. Una carga ideológica para reproducir. Ante ello, observamos dos situaciones: la primera, que el alumno va construyendo la idea de un bien común en el proceso formativo teórico, que es necesario contribuir en la formación del otro, que el otro requiere de un trato especial, dada las condiciones físicas, sociales, escolares y económicas en las que se desenvuelve; la segunda, que a los lugares donde realiza la práctica docente, hay especialistas en el área en que se forma, además, son personas que pudieran contribuir a la formación si les prestaran interés a las actividades que ellos realizan durante la práctica, dado que requieren de la opinión de ellos.

La espera de la aceptación por lo que se hace es parte de saberse acertado y aceptado ante las condiciones en que se realiza la práctica docente como especialista en la educación especial. Ante el discurso que dan los estudiantes se observa la interiorización por el deber ser del educador en educación especial, el estar con el otro y ser parte del otro. A la vez un proceso de incertidumbre de que si lo que se hace en el tiempo que dura la práctica es correcto. En primera instancia porque es aprendiz del oficio, la polivalencia en ellos se va formando. Sin embargo, en este proceso formativo que adquiere en la práctica docente hay una novedad, de que él sabrá qué hacer en las situaciones que se presenten dentro del aula, ello incluye la didáctica, las interacciones dentro del aula y los contenidos del plan de estudio con el que forma a los alumnos con los que trabaja.

La formación como especialista en la educación especial en los estudiantes de acuerdo al plan y programas de estudio con los que se forman, considera que el estudiante debe saber responder a las necesidades educativas que se le 
presenten cuando ejerza la docencia en la educación especial, ante ello, el plan de estudio considera que los estudiantes al realizar practicas docentes en el tiempo que tarda la formación en la escuela normal vaya adquiriendo las habilidades y las capacidades de atender a personas que tienen necesidades educativas especiales.

Ante ello, los estudiantes van haciendo suyo un lenguaje de tecnicismos respecto a algunas necesidades educativas que se presenten en el tiempo que realizan la práctica docente. Por ejemplo en los discursos que dicen, se identifican conceptos como: adecuación, discapacidad intelectual. Este lenguaje incorporado a su profesión, es un discurso de la efectividad del aula dentro de la escuela normal para que el alumno lo apropie. Sin embargo, se da cuenta el alumno que este lenguaje no es vivido en los profesores de las escuelas donde realizan la práctica docente.

Por ejemplo que los estudiantes mencionen que los profesores no hacen adecuaciones, no los apoyan pero sí les exigen material, lo que nos lleva a la siguiente reflexión. Los profesores que deberían fortalecer la formación de los alumnos en la educación especial manifiestan un abandono hacia la práctica docente cuando el alumno practicante llega al aula. Se le deja ser. En este ser que el alumno encuentra en el tiempo de práctica, se da cuenta de cómo funciona el sistema, de cómo fluyen las prácticas docentes de quienes tienen el compromiso de que el plan y programa de estudio con el que se forma en la educación básica se cumpla.

\section{Conclusiones}

El discurso que tienen los alumnos respecto a su formación inicial da cuenta de que se les ha ido formando en el deber, en la responsabilidad de educar. Se les forma un ser en el deber, un compromiso hacia el otro. Sin embargo encuentran en el proceso de la formación que tal deber no se cumple, dado que, la formación teórica que se les provee en la escuela normal no se refuerza con la formación en la práctica docente que realizan semestre tras semestre en el tiempo que dura la licenciatura. En este primer acercamiento que realizamos a la información obtenida en el análisis del discurso hecho, consideramos pertinente una cercanía académica entre escuela normal formadora $y$ escuelas de educación básica que dan espacios para que el profesor en formación inicial realice la práctica docente. Otro elemento que consideramos, se enfoca a repensar la formación en educación especial, desde disciplinas sociológicas y antropológicas.

\section{Bibliografía} Chiapas. 
Barba, B. (2010). Entre maestros: reforma de la formación docente en Aguascalientes. Revista Mexicana de Investigación Educativa, 15 (44), 251-275.

Bourdieu, P., \& Passeron, J.-C. (1996). La reproducción. Elementos para una teoría del sistema de enseñanza. México: Fontamara.

Day, C. (2005). Formar docentes. Cómo, cuándo y en qué condiciones aprende el profesorado. España: Narcea.

Farías-Martínez, G. M., \& Ramírez-Montoya, M. S. (2010). Desarrollo de cualidades reflexivas de profesores en formación inicial a través de portafolios electrónicos. Revista Mexicana de Investigación Educativa , 15 (44), 141-162.

Furlan, A. (2005). Entre las "buenas intenciones" y los "acuerdos funcionales". El tema de la violencia en la formación inicial de profesores de secundaria en México. Revista Mexicana de Investigación Educativa , 10 (27), 1083-1108.

Gimeno, S. J. (2001). Educar y convivir en la cultura global: Las exigencias de la ciudadanía. Madrid: Ediciones Morata.

Ibáñez, J. (2003). Más allá de la sociología. El grupo de discusión: técnica y crítica. Madrid: Siglo XXI.

Morin, E. (2010). ¿Hacia el abismo? Globalización en el siglo XXI. España: Paidós.

Pérez Gómez, Á. I. (1999). La función y formación del profesorado en la enseñanza para la comprensión. Diferentes perspectivas. En \&. G. S. J. Gimeno, Comprender y transformar la enseñanza (págs. 398-429). Madrid: Morata. Secretaría de Educación Pública. (2004). Plan y programa de estudio 2004. Licenciatura en Educación Especial. México: Secretaría de Educación Pública.

Tardif, M. (2004). Los saberes del docente y su desarrollo profesional. España: Narcea.

Torres, J. (1994). Globalización e interdisciplinariedad: El curriculum integrado. Madrid: Ediciones Morata.

Van Dijk, T. A. (2005). Ideología y análisis del discurso (Vol. 10). Maracaibo, Venezuela: Utopía y Práxis Latinoamericana. 